\title{
Analyse Du Remplissage Sédimentaire De La Partie Centrale Du Bassin Des Iullemmeden (Niger Central) Pendant La Période Allant Du Crétacé Supérieur Au Paléocène-Yprésien Et Transgressions Associées
}

\author{
Dr. Karimou Laouali Idi
}

Université Abdou Moumouni, Faculté des Sciences et Techniques, Département de géologie, Laboratoire de Géologie, Equipe Bassins et Géoressources, Niamey, Niger

\section{Dr. Abdoulwahid Sani}

Université d’Agadez, Faculté des Sciences et Techniques, Département de Géologie, Niamey, Niger

\section{Prof. Moussa Konaté}

Université Abdou Moumouni, Faculté des Sciences et Techniques, Département de géologie, Laboratoire de Géologie, Equipe Bassins et Géoressources, Niamey, Niger

Doi:10.19044/esj.2021.v17n29p1

Submitted: 23 June 2021

Accepted: 03 August 2021

Published: 31 August 2021
Copyright 2021 Author(s)

Under Creative Commons BY-NC-ND 4.0 OPEN ACCESS

Cite As:

Laouali Idi K., Sani A. \& Konaté M. (2021). Analyse Du Remplissage Sédimentaire De La Partie Centrale Du Bassin Des Iullemmeden (Niger Central) Pendant La Période Allant Du Crétacé Supérieur Au Paléocène-Yprésien Et Transgressions Associées. European Scientific Journal, ESJ, 17(29), 1.

https://doi.org/10.19044/esj.2021.v17n29p1

\section{Résumé}

L’analyse du remplissage sédimentaire du bassin des Iullemmeden pendant la période allant du Crétacé supérieur au Paléocène-Yprésien a permis de mettre en évidence une succession de 5 à 6 épisodes transgressifs. La succession verticale des faciès, identifiés pour cette période, comprend de la base au sommet : des grès calcaires et argiles à gypses, d'âge Turonien inférieur-Cénomanien supérieur mises en place pendant la transgression T1, des calcaires et argilites gypsifères, d'âge Turonien supérieur déposés au cours de la transgression T2, des calcaires et marnes sableuses, d'âge Sénonien inférieur et moyen rattachés à la transgression T3, des siltites et des argilites, d’âge maastrichtien, mises en place pendant la transgression T4 à Libycoceras 
ismaeli et Laffiteina bibensis et des argilo-calcaires d'âge paléocène-yprésien, associés aux transgressions $\mathrm{T} 5$ et $\mathrm{T} 6$ respectivement à Ranikothalia bermudezi et Lochkartia hamei. Néanmoins, il ressort une controverse dans la datation et le classement des différentes transgressions ainsi mise en évidence dans ce bassin des Iullemmeden. Ainsi, l'objectif principal de la présente étude est de proposer une synthèse des différentes transgressions identifiées dans ce bassin. La méthodologie mise en œuvre est basé sur l'exploitation des données bibliographiques soutenue par des données lithostratigraphiques, paléontologiques, sédimentologiques. Les résultats de cette étude montrent que le bassin des Iullemmeden à été comblé par six ou sept transgressions marines au lieu de cinq ou six reconnues.

Mots clés : Bassin Des Iullemmeden, Crétacé Supérieur, Paléocène-Yprésien, Transgression À Lockartia Haimei

\section{Analysis Of The Central Part Sedimentary Infilling Of The Iullemmeden Basin (Central Niger), During The Period Ranging From Upper Cretaceous To Paleocene-Ypresian And Associated Transgressions}

\section{Dr. Karimou Laouali Idi}

Université Abdou Moumouni, Faculté des Sciences et Techniques, Département de géologie, Laboratoire de Géologie, Equipe Bassins et Géoressources, Niamey, Niger

\section{Dr. Abdoulwahid Sani}

Université d'Agadez, Faculté des Sciences et Techniques, Département de Géologie, Niamey, Niger

\section{Prof. Moussa Konaté}

Université Abdou Moumouni, Faculté des Sciences et Techniques, Département de géologie, Laboratoire de Géologie, Equipe Bassins et Géoressources, Niamey, Niger

\footnotetext{
Abstract

The analysis of the sedimentary infilling of the Iullemmeden basin during the period ranging from Upper Cretaceous to Paleocene-Ypresian has revealed a succession of 5 or 6 transgressive episodes.

The vertical succession of facies, identified for this period, includes from the bottom to top: calcareous sandstones and gypsum clays, of Lower Turonian-Upper Cenomanian age, deposited during the T1 transgression, gypsiferous limestones and mudstones of Upper Turonian deposited during theT2 transgression, sandy limestones and marls of Lower and Middle
} 
Senonian age related to T3 transgression, siltstones and argillites, of Maastrichtian age, deposited during the T4 transgression with Libycoceras ismaeli and Laffiteina bibensis and clay-limestones of Paleocene-Ypresian age, associated with transgressions T5 and T6 respectively with Ranikothalia bermudezi and Lochkartia hamei. Nevertheless, a controversy emerges in the dating and the classification of the various transgressions thus highlighted in this basin of the Iullemmeden.

Thus, the main objective of this study is to provide a synthesis of the various transgressions identified in this basin. The methodology implemented is based on the use of bibliographic data supported by lithostratigraphic, paleontological and sedimentological data. The results of this study show that the Iullemmeden basin was infilled by six or seven marine transgressions instead of five or six recognized.

Keywords: Iullemmeden Basin, Upper Cretaceous, Paleocene-Ypresian, Lockartia Haimei Transgression

\section{Introduction}

Le bassin des Iullemmeden, de forme pentagonale (Radier, 1953) est un vaste bassin intracratonique qui s'étend au Sud du Hoggar. Il couvre la majeure partie du territoire de la république du Niger et à s'étend en Algérie, au Mali, au Bénin et au Nigéria. Son histoire géologique commence dès le Cambrien dans le synclinal de Tin Séririne, en Algérie (Joulia, 1959). Par la suite, les aires de sédimentation se sont déplacées vers le Sud, déplacement se traduisant par la mise en place des formations margino-littorales et continentales (Bellion, 1987). Pendant la période allant du Cénomanien inférieur au Sénonien inférieur et moyen, une sédimentation détritique engendrée par les trois premières transgressions marines (T1, T2 et T3) a contribué au remplissage du bassin des Iullemmeden. Cet ensemble détritique est recouvert par la quatrième transgression à Libycoceras et Laffiteina d'âge maastrichtien avec 2 fluctuations : (1) La première correspond à la formation d'Alanbanya (Lower sandstones) et à la formation du Farin Doutchi (Mosasaurus shales ou formation de Dukamaje au Nigéria) et (2) la deuxième fluctuation correspond à la formation d'In Wagar (Upper Sandstones et Mudstones ou formation de Wurno au Nigéria) (Greigert, 1966; Dikouma, 1990).

Au-dessus de ces dernières formations reposent les dépôts de la formation de Garadaoua, d'âge paléocène-yprésien, constituée des argilocalcaires et des schistes papyracés mises en place par la transgression marine T5 (Greigert, 1966 ; Boudouresque et al., 1982; Dikouma, 1990). Du Crétacé supérieur au Paléocène-Yprésien, cinq transgressions ont été identifiées (Greigert, 1966; Boudouresque et al., 1982). L'objectif de cette étude est 
d'apporté une précision sur les transgressions du Crétacé supérieur et du Paléocène-Yprésien sur la base des récents travaux (Laouali Idi, 2020) et l'exploitation des données issus des travaux antérieurs (Furon, 1935; Greigert, 1966; Boudouresque et al., 1982; Dikouma et al., 1993;) dans la partie nigérienne de ce bassin des Iullemmeden. La présente étude a porté sur la partie centrale du bassin notamment à Garadaoua. Elle s'est focalisée sur les observations lithostratigraphiques, paléontologiques, sédimentologiques et structurales. Cela a permis de discuter de l'âge des transgressions ainsi que de leur nombre. Aussi, l'analyse de l'ensemble des données obtenues a permis de proposer une reconstitution paléogéographique du bassin des Iullemmeden entre le Crétacée supérieur et le Paléogène en relation avec l'ouverture de l'Atlantique sud.

\section{Contexte géologique du bassin des Iullemmeden}

Le bassin des Iullemmeden est constitué d'une alternance de sédiments marins et de dépôts continentaux mis en place lors des différents épisodes transgressifs et régressifs qui ont marqué son histoire géologique.

\section{Présentation du bassin des Iullemmeden}

Le bassin des Iullemmeden est limité au Sud par le bouclier BéninoNigérian, au Sud-ouest par le massif cristallin du Liptako, au Nord-ouest par 1'Adrar des Iforas, au Nord par le Hoggar, au Nord-Est par les massifs de l'Aïr et à l'Est par le Damagaram-Mounio (Figure 1).. Il communique au NordOuest avec le bassin de Taoudenni par le détroit de Gao et à l'Est avec le bassin du Niger Oriental par le seuil du Damergou (Bellion, 1989) (Figure 1).

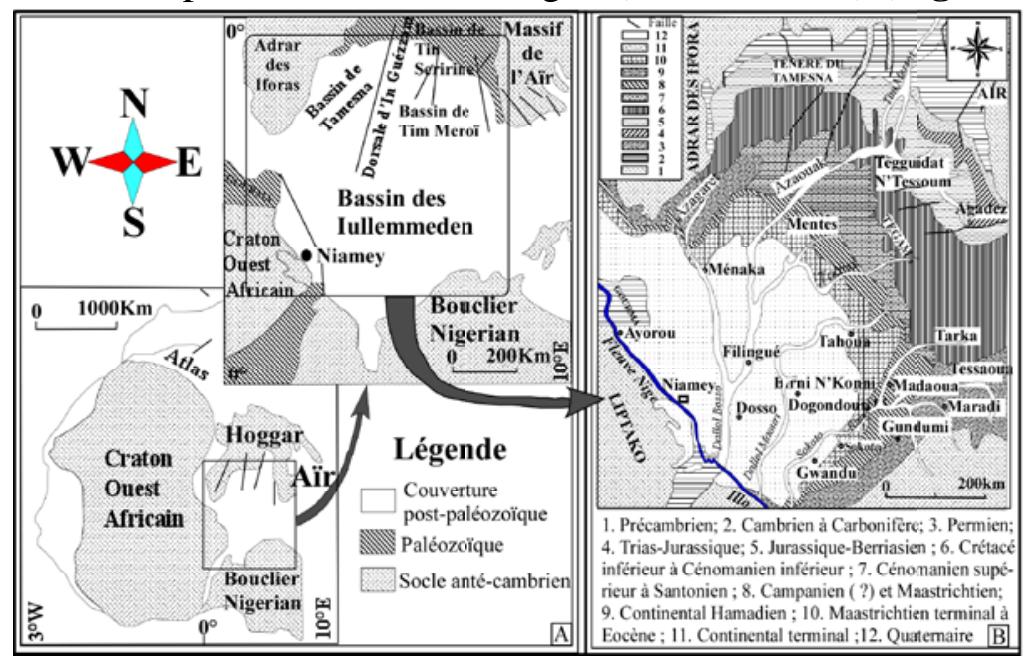

Figure 1. Carte géologique du bassin des Iullemmeden. A) Carte de situation du bassin des Iullemmeden (Wright et al. 1993). B) Carte géologique schématique du bassin des Iullemmeden (Greigert et Pougnet, 1965) 
Ce bassin des Iullemmeden s'étend du Nord au Sud sur une longueur d'environ $1000 \mathrm{~km}$ et d'Est en Ouest sur une largeur d'environ $980 \mathrm{~km}$. Sa superficie est estimée à $525000 \mathrm{~km}^{2}$, dont environ $31000 \mathrm{~km}^{2}$ au Mali, 317 $000 \mathrm{~km}^{2}$ au Niger, $60000 \mathrm{~km}^{2}$ au Nigéria, $10000 \mathrm{~km}^{2}$ au Benin et 106549 $\mathrm{km}^{2}$ en Algerie (Laouali Idi et al., 2019). Dans la partie centrale du bassin, correspondant à la région de Doutchi, l'épaisseur maximale du remplissage sédimentaire est d'environ 2000 m (Greigert, 1966 ; Abdou Ali, 2018).

Le bassin des Iullemmeden correspond à un domaine de sédimentation intracratonique (Figure 1) (Bertrand-Sarfati et al., 1977) affecté par des mouvements épirogéniques (Faure, 1966) qui auraient favorisé une migration des aires de dépôts vers le Sud-Ouest pendant le Mésozoïque et le Paléogène. En conséquence, le remplissage sédimentaire est essentiellement constitué par des dépôts paléozoïques au Nord et méso-cénozoïques au Sud (Figure 2). En effet, les formations paléozoïques affleurantes dans la partie Nord de ce bassin sont essentiellement reparties entre les sous-bassins de Tin Séririne, de Tamesna et de Tim Mersoï, ainsi qu’à son extrémité Sud-Ouest, englobant les sous-bassins de Kandi au Benin et de Sokoto au Nigeria, (Bellion, 1989). Les sédiments mésozoïques occupent la plus grande partie du bassin. Les formations cénozoïques reposent en discordance de ravinement sur les formations mésozoïques (partie centrale du bassin) et infracambriennes (bordure Est du Craton Ouest Africain) et en discordance majeure sur les formations panafricaines et paléoprotérozoïques (bordure Ouest et Sud du bassin) (Greigert, 1966 ; Laouali Idi, 2020) (Figure 2).

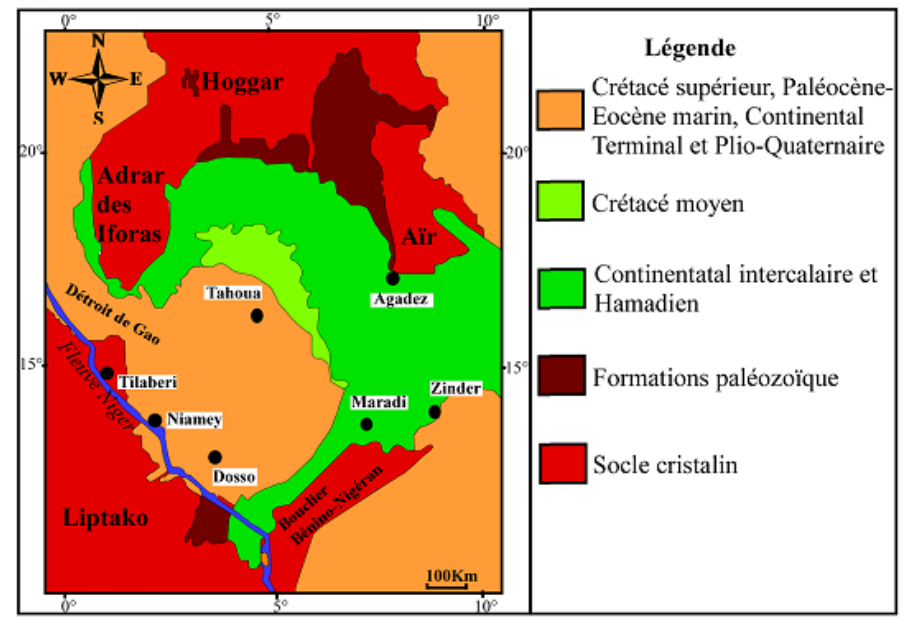

Figure 2. Carte géologique simplifiée du bassin des Iullemmeden (Greigert, 1966, modifié)

Cadre stratigraphique et paléogéographique du bassin des Iullemmeden Stratigraphie du bassin des Iullemmeden

D'après Kilian (1931), l'histoire géologique du bassin des Iullemmeden est caractérisée par deux périodes de transgression marine 
séparées par une longue période de régression allant du Permien au Crétacé inférieur. Selon Joulia (1963) ce processus de transgression et de régression a mis en évidence trois incursions marines qui se sont produites pendant le Paléozoïque (le Silurien, le Dévonien moyen et supérieur et le Carbonifère inférieur), auxquelles il faut ajouter les cinq incursions marines du MésoCénozoïque (Greigert, 1966 ; Kogbé et Lang, 1994; Laouali Idi, 2020) (Figures 3 et 4). Aussi, la sédimentation du bassin des Iullemmeden est marquée par plusieurs alternances de dépôts marins et de sédiments continentaux dont les limites spatiales et temporelles, selon Boudouresque (1980), ne sont pas toujours faciles à déterminer avec précision (Figure 3).

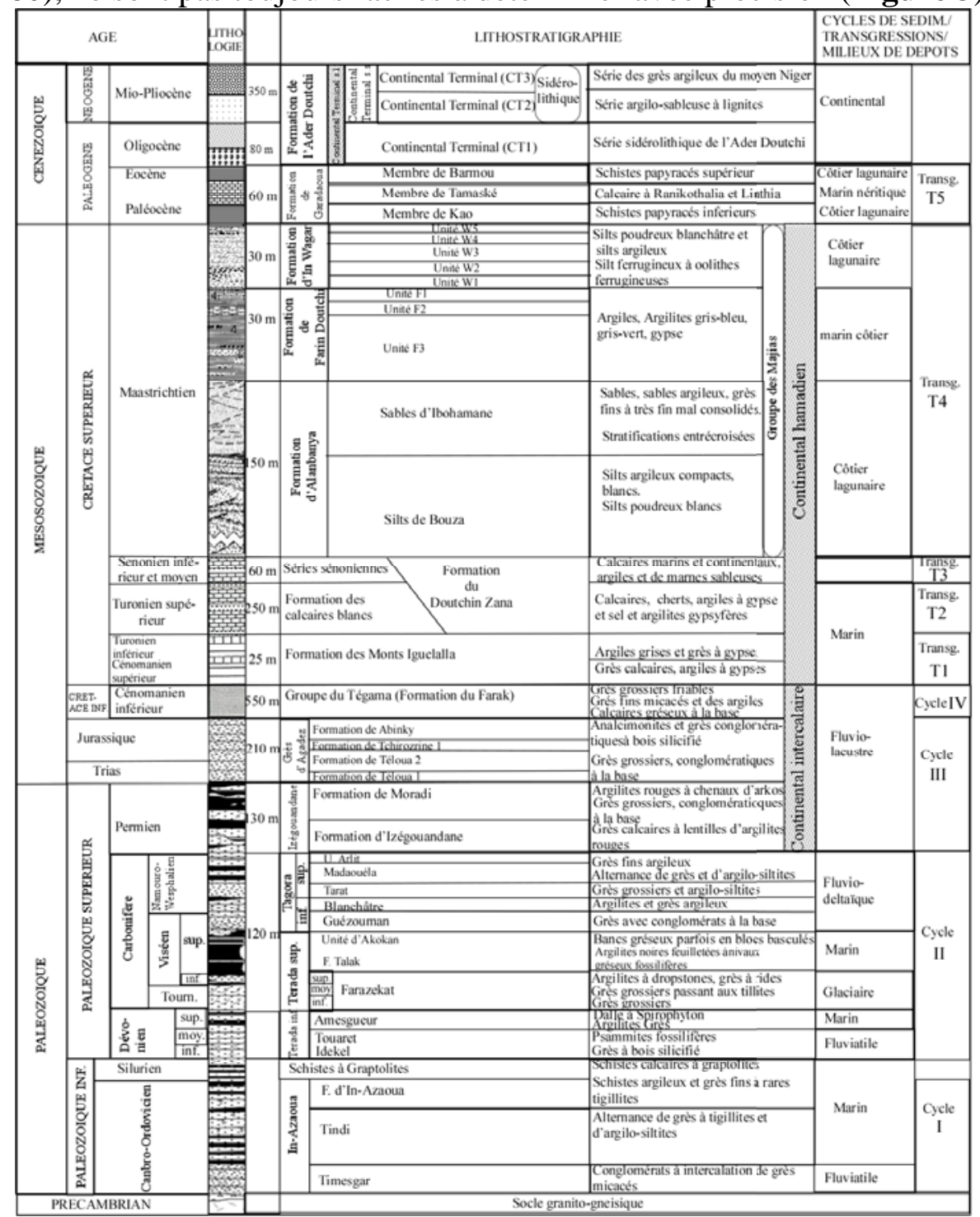

Figure 3. Colonne lithoshtratigraphique du bassin des Iullemmeden (Greigert, 1966; Taquet, 1976; Hanon, 1984, modifié) 


\section{Paléogéographique du bassin des Iullemmeden}

Du Crétacé supérieur (Cénomanien supérieur-Maastrichtien) au Paléogène (Paléocène-Yprésien), le bassin des Iullemmeden a connu cinq cycles transgressifs successifs notés T1, T2, T3, T4 et T5 (Greigert, 1966) (Figure 4). Les différents épisodes transgressifs sont séparés par des épisodes régressifs (Greigert, 1966 ; Boudouresque et al., 1982 ; Dikouma, 1990) (Figure 4). Il s'agit des transgressions T1 et T2 respectivement à Néolobites et Nigericeras du Cénomanien supérieur à Turonien inferieur, des transgressions T3 et T4 à Libycoceras ismaeli et Laffiteina bibensis, datée du Maastrichtien supérieur et de la transgression T5 à Ranikothalia et Lockhartia, datée du Paléocène-Yprésien (Dikouma, 1990) (Figure 4).

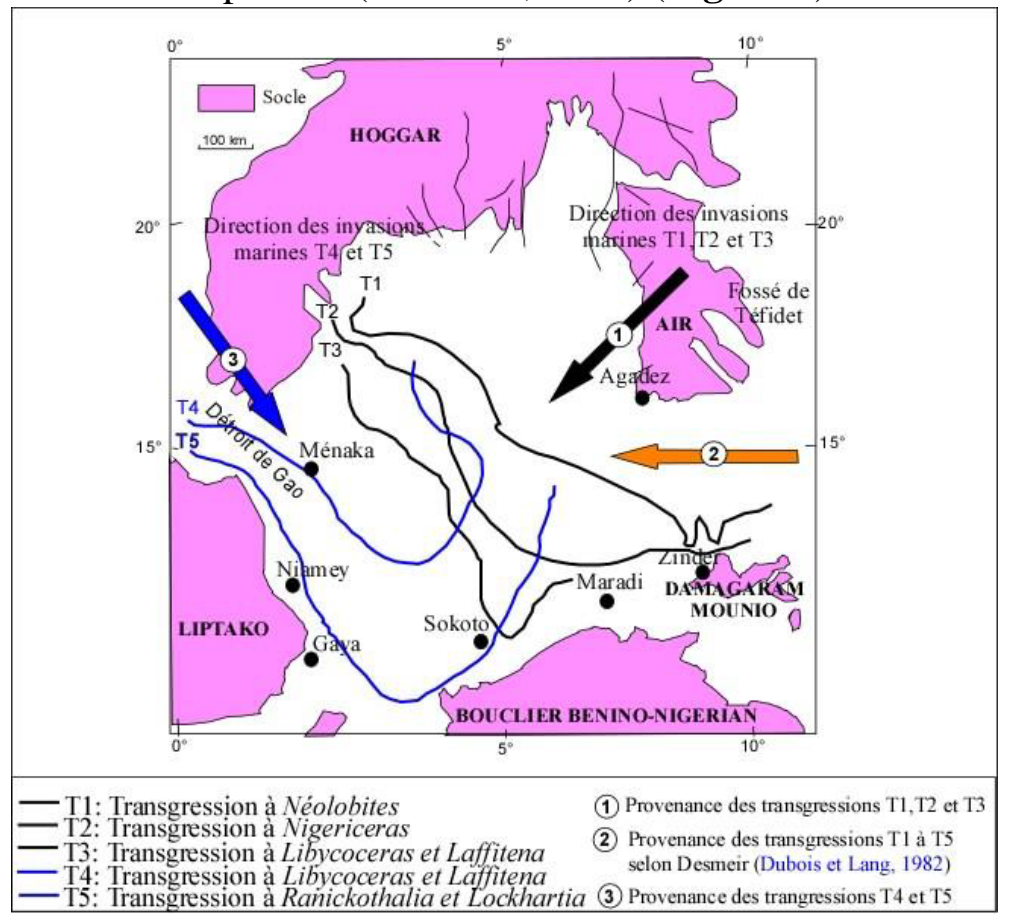

Figure 4. Extension des transgressions crétacées et tertiaires dans le bassin des Iullemmeden (Greigert, 1966; Kogbé et Lang, 1994 ; Miko, 1999, modifié)

Dikouma et al. (1993) considèrent la position de la mer avant la transgression T1 de Greigert (1966), comme étant la première transgression dans le bassin des Iullemmeden. Ce qui a amené ces auteurs à définir six transgressions (Figure 5): (i) avant la transgression à Neolobites et Nigericeras du Cénomanien inférieur T1, la position de la mer a été définie; (ii) les transgressions à Neolobites et Nigericeras du Cénomanien supérieur sont désignées par T2 et T3; (iii) les transgressions successives à Libycoceras et Laffiteina du Maastrichtien supérieur correspondent à T4 et T5 et (iv) la dernière transgression du Paléocène supérieur est désignée par T6. 


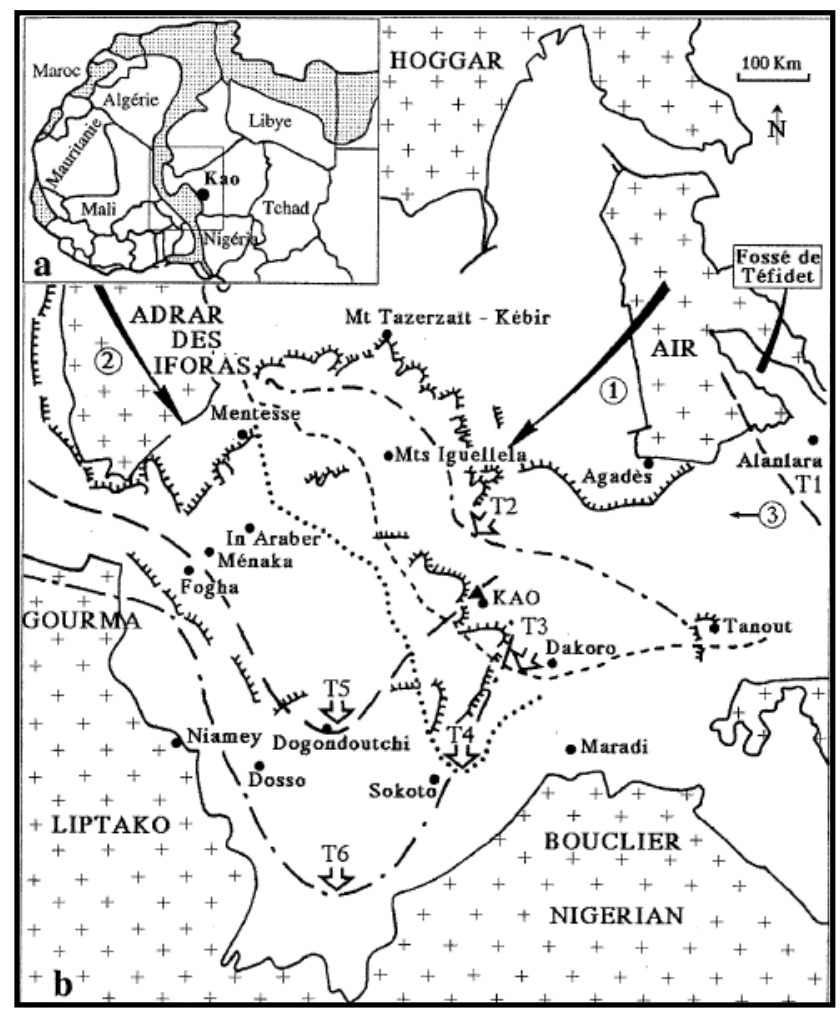

Figure 5. Extension des transgressions crétacées et tertiaires dans le bassin des Iullemmeden. (Greigert 1966, T1 à T6; Lang \& Kogbe, 1994). T1. Mer avant la transgression à Neolobites et Nigericeras. T2 et T3. Transgressions à Neolobites et Nigericeras avec 2 avancées du Cénomanien supérieur-Turonien inférieur. T4 et T5. Transgressions successives (deux fluctuations d'une seule transgression) à Libycoceras du Maastrichtien supérieur (Dikouma et al,. 1993). T6. Transgression du Paléocène supérieur.

(1) Provenance des transgressions cénomano-turoniennes T2 et T3. (2) Provenance des transgressions maastrichtiennes T4 et T5. (3) Provenance des transgressions T4 et T5

(Dubois \& Lang,1981)

\section{Matériel et Méthodes}

L'approche méthodologique adoptée dans cette étude comprend deux étapes : l'exploitation des données bibliographiques et l'analyse sédimentologique (Figure 6).

\section{Exploitation des données bibliographiques}

La compilation des documents disponibles (articles, thèses, rapports, cartes), relatifs à la géologie du bassin des Iullemmeden et de l'Afrique de l'Ouest, a permis de faire un état des lieux sur les caractéristiques des différentes transgressions ayant contribué au remplissage du bassin des Iullemmeden. Les différents documents cartographiques ont été complétés avec les données de terrain. 


\section{Analyse sédimentologique}

L'analyse sédimentologique a été réalisée au niveau des différentes formations marquant la limite Crétacé-Tertiaire. Il s'agit des formations d'In Wagar et de Garadaoua. La série méso-cénozoïque constitue une série complète qui s'étend du Maastrichtien jusqu'à l'Yprésien. Cette série est représentée par des formations silto-argileuses et carbonatées. Soixante-trois (63) échantillons de roches et six-cent cinquante fossiles (650) ont été prélevés. L'échantillonnage a concerné tous les affleurements étudiés. Pour la réalisation des lames minces seuls les échantillons prélevés sur l'affleurement de Garadaoua ont été analysés car ils sont les plus complets. Ainsi, la compilation des données bibliographiques et les données de terrain a permis de dresser une synthèse lithostratigraphiques, paléontologiques, paléogéographique, sédimentologiques et structurales.

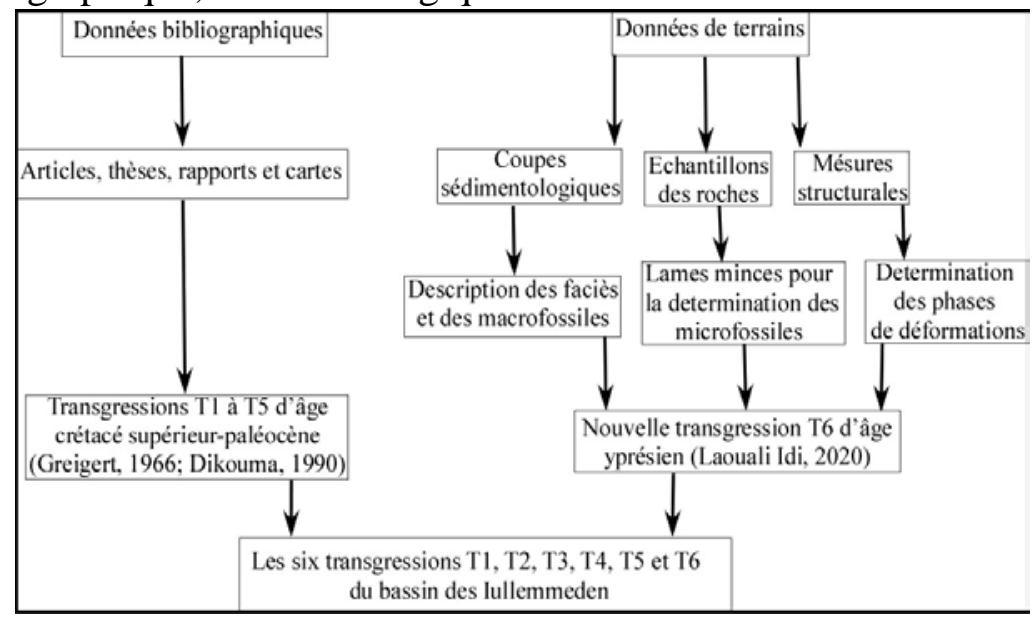

Figure 6. Organigramme de l'approche méthodologique

\section{Résultat}

\section{Analyse sédimentologique}

L’analyse sédimentologique a consisté à l'analyse de la coupe de Garadaoua. Cette coupe regroupe trois formations à savoir : la formation d'In Wagar, la formation de Garadaoua et la formation de l'Ader Doutchi.

- La formation d'In Wagar (W), d'une épaisseur d'environ six (6) mètres, regroupe trois unités :

o L'unité W3 qui est constituée de quatre niveaux (Figure 7). Le niveau de base est représenté par des bancs sableux fins à litages obliques (Figure 8a) arqués ou en auges passant verticalement à des bancs sableux massifs (Figure 7). Les dépôts supérieurs de l'unité W3 sont représentés par des sables fins blanchâtres à litages ondulés (Figure 8c) relayés au sommet par des bancs sableux bioturbés à litages subhorizontaux 
(Figures 7 et 8b). L'unité W3 de la formation d'In Wagar a été attribuée à un talus de progradation d'un front deltaïque (Figure 9).

o L'unité W4, d'environ $10 \mathrm{~m}$ d'épaisseur, comprend sept niveaux (Figures 7 et 8). Le niveau de base est constitué de grès fins argileux beiges (Figure 8d), relayés verticalement par des argilites puis par des argilites silteuses, surmontées par des grès fins argileux plus ou moins schisteux. Le niveau intermédiaire de l'unité W4 est représenté par des grès fins argileux à concrétions calcaires (Figure 8e), qui sont surmontés par des grès fins argileux plus ou moins ferrugineux, à nodules calcaires (Figure 8f). Le sommet de l'unité W4 correspond à des grès moyens argileux, plus ou moins ferrugineux (Figure 8g).

- La formation maastrichtienne d'In Wagar est surmontée par la formation paléocène-yprésienne de Garadaoua. Cette dernière est subdivisée en quatre membres. Il s'agit, de la base au sommet, des membres de : Kao, Tamaské et Barmou tous reconnus par Dikouma (1990) et le membre de Keita mis en évidence par la présente étude. Les quatre membres de la formation de Garadaoua ont été subdivisés en huit niveaux.

o Le premier membre G1 de Kao est constitué de deux niveaux (Figures 7 et 8). Le niveau de base est représenté par des argilites jaunâtres (Figure 8h), très fossilifères, comprenant des nautiles, des échinides (genre Linthia), des bivalves et des gastéropodes (Dikouma, 1990). Tandis que le niveau supérieur est essentiellement constitué de schistes papyracés (Figures $\mathbf{7}$ et 8i). Ce membre correspond à l'épisode régressif paléocène.

o Le second membre de Tamaské (G2) comprend trois niveaux calcaires, d'épaisseur variable, formant des falaises aux bordures rectilignes (Figures 7 et 8). Le niveau de base est constitué de calcaires fossilifères plus ou moins argileux (Figure 8j). Le niveau intermédiaire de ce membre G2 est représenté par des calcaires massifs riches en fossiles (Figure 8k). Le sommet de G2 correspond à des calcaires crayeux et noduleux (Figures $\mathbf{7}$ et $\mathbf{8 n}$ ). Cet ensemble représente l'épisode transgressif paléocène.

o Le troisième membre de Barmou G3 est représenté par un niveau de schistes papyracés ou schistes cartons (Figures 7 et 8o). Ce membre correspond à l'épisode régressif yprésien.

o Le quatrième membre G4 de Keita comprend deux niveaux : le premier marneux, gréseux à tendance conglomératique (Figure 8p) associé à l'épisode transgressif yprésien, et le second est constitué de schistes papyracés (Figure 8q) qualifié de l'épisode régressif ypresien. Le niveau marneux renferme une faune de bivalves, de foraminifères et d'ostracodes (Figures 7 et 8). 
- Le sommet de la coupe de Garadaoua est relayé par la formation de l'Ader Doutchi (Figures 7 et 8). Cette dernière est constituée essentiellement d'argilites bariolées et de siltites argileuses à la base. La formation de l'Ader Doutchi montre une alternance de bancs irréguliers, constitués d'argiles kaoliniques, plus ou moins latéritiques et d'oolithes ferrugineuses dans sa partie médiane. Le sommet de cette formation est constitué de grès à oolithes ferrugineuses (Figure 8r).

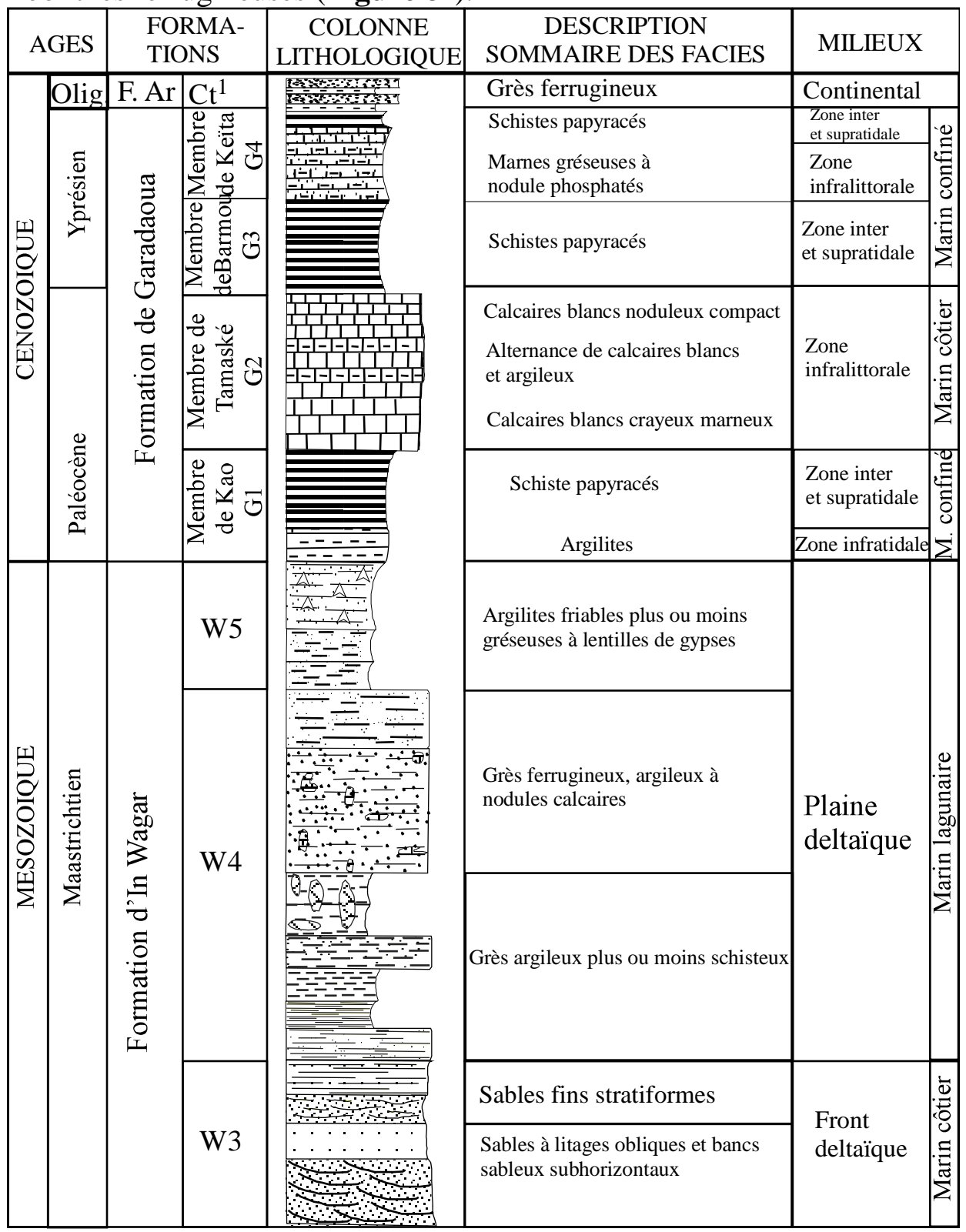

Figure 7. Coupe sédimentologique de Garadaoua 


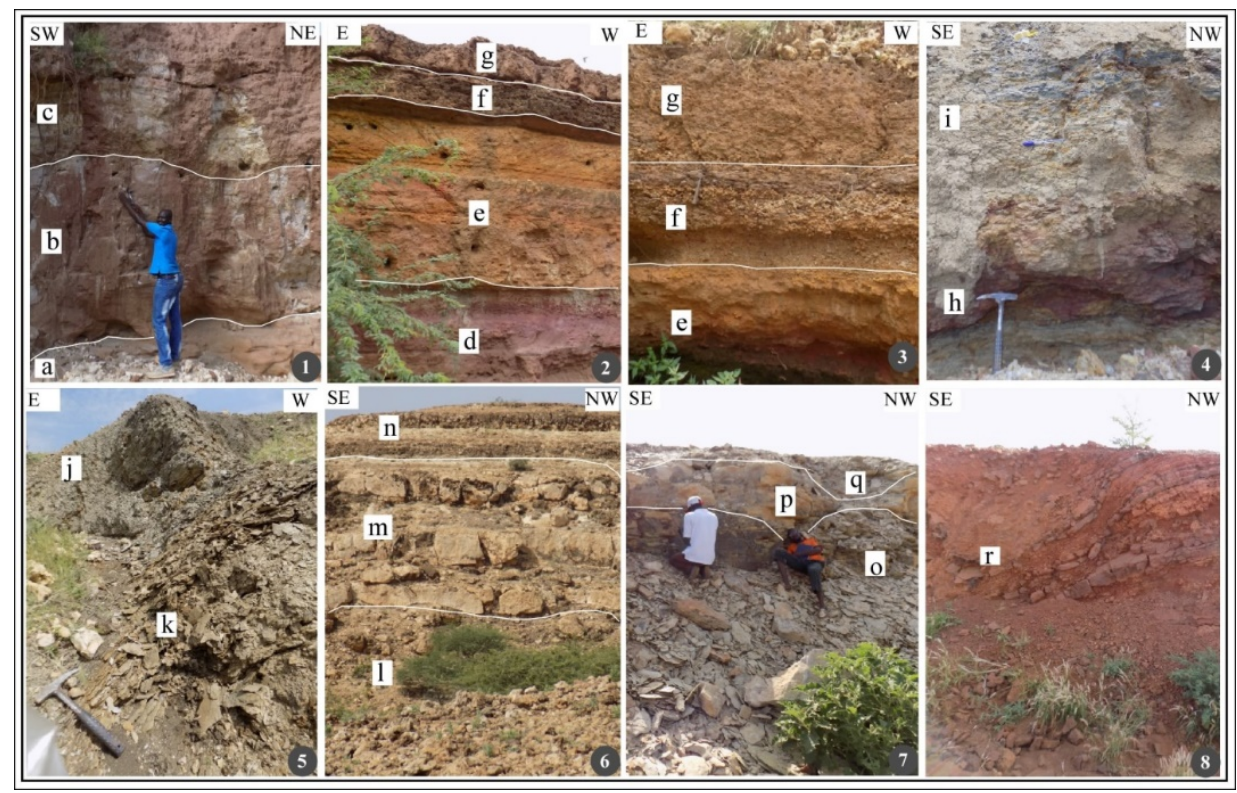

Figure 8. Vue des lithofaciès dominants le long de la coupe de Garadaoua.

1) a) banc sableux à litages obliques b) banc sableux subhorizontal c) Sables fins stratiformes $\mathbf{2}$ et 3) d) argiles schisteuses grises, violets à beiges e) grès fins argileux à concrétions calcaires f) grès fins ferrugineux à nodules calcaires g) grès moyens ferrugineux à nodules calcaires 4) h) argile schisteuse feuilletée i) argilites à lentilles de gypse 5) j) marnes jaunâtres très fossilifères k) schistes papyracés inférieure 6) l) calcaires blancs crayeux et marneux à la base $\mathrm{m}$ ) alternance de calcaires blancs et de calcaires argileux $n$ ) calcaires noduleux compact 7) o) schistes papyracés p) marne gréseuses à nodules phosphatés q) schistes papyracés 8) r) grès ferrugineux

\section{Paléogéographie et environnements de dépôts du bassin des Iullemmeden}

Le schéma paléogéographique du bassin des Iullemmeden du Crétacé supérieur et au Paléocène-Yprésien est marqué par la présence d'une mer épicontinentale, peu profonde, avec en bordure une plaine côtière marécageuse, parsemée de lagunes littorales (Figure 9). Comme évoqué précédemment, du Crétacé supérieur au Paléocène supérieur, cinq transgressions (T1, T2, T3, T4 et T5) ont été identifiées (Boudouresque et al., 1982 ; Dikouma, 1990).Une autre transgression a été mise en évidence par la présente étude.

\section{Les transgressions Maastrichtiennes}

Le Maastrichtien du bassin des Iullemmeden est marqué par deux transgressions marines (Figures 9 et 10). Il s'agit de la transgression T3 à Libycoceras et la transgression T4 à Laffiteina avec 2 fluctuations. La première correspond aux formations d'Alanbanya (Lower sandstones and mudstones ou formation de Taloka au Nigéria) et de Farin Doutchi (Mosasaurus shales ou formation de Dukamaje au Nigéria) et la deuxième 
fluctuation correspond à la formation d'In Wagar (Upper Sandstones et Mudstones ou formation de Wurno au Nigéria) (Dikouma, 1990). A la même période se réalise à l'Ouest, la communication entre le bassin des Iullemmeden et le bassin de Taoudenni à la suite d'un affaissement de direction NW-SE (fossé de Gao) (Greigert, 1966). D'après Guiraud et al. (1981), le système de fracture NW-SE des dépôts maastrichtiens a été à la base de la compression fini-crétacée $\mathrm{N} 130^{\circ}$

Les régions étudiées (Madaoua, de Bouza, de Malbaza et de Keita) constituent l'extension maximale de la deuxième transgression à Libycoceras ismaeli et Laffitteina bibensis du Maastrichtien supérieur. Le littoral correspond à une plaine alluviale à sédimentation siliciclastique fine, avec localement la formation d'un mini-delta (Dikouma, 1990) (Figure 9 A et B). Les alternances silto-sableuses (barres distales) et les corps sableux lenticulaires (barres d'embouchure) forment, avec les sables à litages obliques et horizontaux, le front deltaïque. Les niveaux ferrugineux à racines et à fruits ferruginisés (paléosols) sont assimilés à la plaine deltaïque (Figure 9 B). D’après Dikouma (1990) la présence de ces fruits, dont certains pourraient correspondre à des Rhizophoracées (plantes des mangroves) ainsi que la présence de natrojarosites $(\mathrm{NaFe}(\mathrm{OH}) 6(\mathrm{SO} 4) 2$ dans les paléosols, suggère un environnement de type fluvio-deltaïque (mangrove, lagunaire ou tout au moins marécageux). La présence des niveaux ferruginisés et phosphatés (Unité W4) indique que la région a subi une importante ferruginisation et phosphatisation. Ces phénomènes de ferruginisation et phosphatisation ont été signalé au Sénégal par Flicoteaux (1976). Ils résulteraient d'une altération lithodépendante sous climat tropical chaud et humide après émersion des sédiments. Cette idée de climat tropical chaud et humide a été confirmée par Boudouresque (1980).

\section{Les transgressions paléocène-yprésiennes}

Après les transgressions marines maastrichtiennes (T1, T2, T3 et T4), la région a subi une nouvelle incursion marine au Paléocène (T5). Tout comme la transgression T4, l'incursion marine paléocène (T5) a atteint le bassin des Iullemmeden par le fossé de Gao (Figure 9 C). Pendant le Paléocène, le domaine marin est caractérisé par une sédimentation de type plateforme carbonatée (Figure 9 C). La zone littorale à sédimentation argilo-siltosableuse est occupée par une mangrove parsemée de lacs ou de lagunes (Dikouma, 1990). Après le maximum transgressif qui correspond à la mise en place du membre de Tamaské, un épisode régressif a entraîné un recul momentané de la mer associé à la mise en place du membre de Barmou. Après cet épisode régressif, la mer revient à nouveau à l'Yprésien (Figure $9 \mathrm{E}$ et F). Cette brève remontée du niveau de la mer représente la transgression à Lockhartia hamei (T6). Les dépôts marneux et schisteux (schistes papyracées) 
correspondants aux membres de Keita, liés à la transgression T6, ont été mis en place pendant l’Yprésien. Ces dépôts éocènes sont surmontés par la série sidérolithique de l'Ader Doutchi constituées de sables et d'argilites kaoliniques ferruginisées.

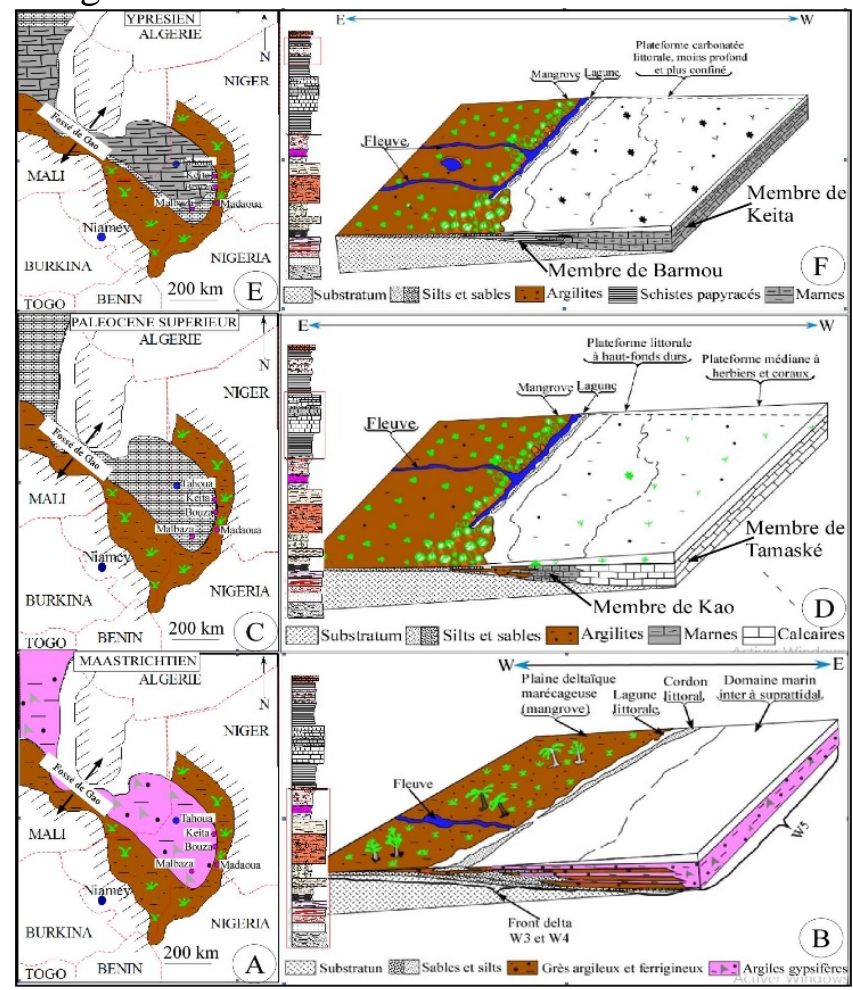

Figure 9. Schéma paléogéographique du bassin des Iullemmeden pour la période allant du Maastrichtien à l’Yprésien (Petters, 1978; Dikouma, 1990; modifié). A) Provenance de la transgression maastrichtienne T4. B) Bloc diagramme montrant les différents environnements sédimentaires maastrichtiens et leur organisation dans la zone d'étude C) Provenance de la transgression paléocène T5. D) Bloc diagramme montrant les différents environnements sédimentaires paléocènes et leur succession dans la zone d'étude. E) Provenance de la transgression yprésienne T6. F) Bloc diagramme montrant les différents environnements sédimentaires yprésiens et leur disposition dans la zone d'étude

\section{Discussion sur les transgressions méso-cénozoïques dans le bassin des Iullemmeden}

Au Crétacé supérieur, le golfe mésogéen, existant déjà à l'Est de l'Aïr, pénètre dans le Niger occidental. Il est à l'origine de la mise en place de séries sédimentaires où alternent des dépôts marins (calcaires et marnes plus ou moins gréseuses à faunes mésogéennes de mers chaudes épicontinentales) et des dépôts subcontinentaux (argilites et grès riches en végétaux et en restes de vertébrés) (Dikouma et al. 1993). Le classement des transgressions dans le bassin des Iullemmeden fait l'objet de controverse. Pour certains auteurs, il y’aurait cinq (5) épisodes transgressifs (Greigert, 1966) tandis que pour 
d'autres, il y'aurait six (6) transgressions (Dikouma et al. 1993), notées T1, T2, T3, T4, T5 et T6. Leur classement et leur âge varient selon les auteurs. Les premiers auteurs, Greigert (1966), Petter (1978), Dubois et Lang (1981), Alzouma (1982), Hanon (1984), Miko (1999) considèrent un âge cénomanien supérieur à la première transgression T1 à Neolobites et Nigericeras, alors que Dikouma et al. (1993) et Favreau (2000) lui attribuent un âge cénomanien inférieur (Figure 10). Les transgressions T2 et T3 classement de Greigert (1966), datant du Turonien inférieur (T2) et du Maastrichtien (T3), correspondent respectivement à celles du Cénomanien supérieur et du Turonien inférieur décrites par Mathey et al. (1991) et Dikouma et al. (1993) (Figure 10). Tandis que les transgressions T4 et T5 rattachées par Greigert (1966) respectivement au Maastrichtien-Danien et au Paléocène supérieur auraient plutôt un âge maastrichtien supérieur pour Dikouma et al. (1993) (Figure 10). Ainsi, la transgression T6 du Paléocène supérieur selon Dikouma et al. (1993) correspondrait quant à elle à la transgression T5 de Greigert (1966).

Dans le cadre de la présente étude, la mise en évidence d'un niveau marneux, d'environ $1 \mathrm{~m}$ d'épaisseur, riche en microfossiles de foraminifères de l'espèce Lockhartia hamei, au-dessus des schistes papyracées supérieurs (membre de Barmou), a conduit à distinguer une nouvelle transgression T6, d’âge yprésien (Greigert, 1966) ou T7 (Dikouma et al., 1993), selon la classement considéré (Figure 10).Partant du classement de Greigert (1966), Petter (1978), Dubois et Lang (1981), Alzouma (1982), Hanon (1984), Miko (1999), cette étude met en évidence six (6) transgressions marines (T1, T2, T3, T4, T5 et T6) (Figure 10) dans le bassin des Iullemmeden du Crétacé supérieur au Paléogène.

\begin{tabular}{|c|c|c|c|c|c|c|c|c|c|}
\hline \multirow{2}{*}{$\begin{array}{l}\text { Systèmes } \\
\text { Etapes }\end{array}$} & \multicolumn{9}{|c|}{ NIGER } \\
\hline & \multicolumn{3}{|c|}{ Greigert (1966) } & \multicolumn{2}{|c|}{ Greigert et Pougnet (1967) } & \multicolumn{2}{|l|}{ Dikouma et al. (1993) } & \multicolumn{2}{|l|}{ Présente étude } \\
\hline $\begin{array}{l}\text { Pliocène } \\
\text { Miocène }\end{array}$ & \multicolumn{3}{|c|}{ Continental terminal } & & & \multirow{2}{*}{\multicolumn{2}{|c|}{$\begin{array}{l}\text { Formation de } \\
\text { Birni N'Konni }\end{array}$}} & \multirow{2}{*}{\multicolumn{2}{|c|}{ Grès ferrugineux $\mathrm{Ct}^{1}$ ) }} \\
\hline \multirow{2}{*}{$\begin{array}{l}\text { Eocène } \\
\text { (Yprésien) }\end{array}$} & & & & \multirow{2}{*}{\multicolumn{2}{|c|}{ 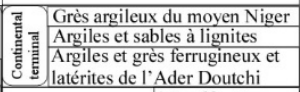 }} & & & & \\
\hline & Schistes pa & apyracés & & & & \multicolumn{2}{|l|}{$\begin{array}{l}\text { Formation de } \\
\text { l'Ader Doutchi }\end{array}$} & \begin{tabular}{|l|} 
Schistes papyracés \\
Marnes \\
Schistes papyracés
\end{tabular} & \\
\hline \multirow[t]{2}{*}{ Paléocène } & \multicolumn{2}{|c|}{ Calcaires et marnes } & \multirow[b]{2}{*}{ T5 } & \multirow{2}{*}{$\begin{array}{l}\text { Schistes papyracés } \\
\text { êt calcaires et } \\
\text { marnes blancs }\end{array}$} & \multirow{2}{*}{$\begin{array}{l}\text { à Lockhartia } \\
\overline{a ̀} \text { Ōperculonoides } \\
\text { (Ranikothalia) }\end{array}$} & \multirow{2}{*}{ 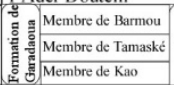 } & \multirow{2}{*}{ T6 } & Schistes papyracés & \\
\hline & \multicolumn{2}{|c|}{ Schistes papyracés } & & & & & & $\begin{array}{l}\text { Calcaires et marnes } \\
\text { Schistes papyracés }\end{array}$ & T5 \\
\hline \multirow{4}{*}{$\begin{array}{l}\text { Sénonien } \\
\text { supérieur }\end{array}$} & \multirow{2}{*}{\multicolumn{2}{|c|}{$\begin{array}{l}\text { Calcaires à Libycoceras } \\
\text { Grès et argiles }\end{array}$}} & & \multirow{4}{*}{\begin{tabular}{|l|} 
Formation d'In Wagar \\
Formation de Farin Doutchi \\
Formation de I'Alanbanya \\
\end{tabular}} & \multirow{2}{*}{ T5 } & Argilites grèseuses & \\
\hline & & & & & & & & Sables fins et argilites & \\
\hline & \multirow{2}{*}{\multicolumn{2}{|c|}{$\begin{array}{l}\text { Calcaires à Libycoceras } \\
\text { Grès et argiles }\end{array}$}} & & & & & & Calcaires à Libycoceras & \\
\hline & & & & & & & T4 & Grès et argiles & \\
\hline $\begin{array}{l}\text { Sénonien } \\
\text { inférieur } \\
\text { et moyen }\end{array}$ & \multicolumn{2}{|c|}{$\begin{array}{l}\text { Calcaires marins et } \\
\text { continentaux } \\
\text { (attapulgite) }\end{array}$} & & \multicolumn{2}{|c|}{ Calcaires et argiles vertes de Zakak } & \multicolumn{2}{|l|}{ Séries sénoniennes } & & \\
\hline $\begin{array}{l}\text { Turonien } \\
\text { supérieur }\end{array}$ & $\begin{array}{l}\text { Série des } \\
\text { calcaires } \\
\text { blancs }\end{array}$ & $\begin{array}{l}\text { Argiles } \\
\text { calcaires } \\
\text { (attapulgites) }\end{array}$ & & \multicolumn{2}{|c|}{ Calcaires blancs } & $\begin{array}{l}\text { Formation des } \\
\text { calcaires blancs }\end{array}$ & & & \\
\hline \multirow{2}{*}{$\begin{array}{l}\text { Turonien } \\
\text { inférieur } \\
\text { Cénomanien } \\
\text { supérieur }\end{array}$} & \multirow{2}{*}{$\begin{array}{l}\text { Série } \\
\text { des Monts } \\
\text { Iguellala }\end{array}$} & $\begin{array}{l}\text { Calcaires à } \\
\text { Nigericeras }\end{array}$ & $\mathrm{T} 2$ & \multirow{2}{*}{$\begin{array}{l}\text { Grès et argiles } \\
\text { glauconieux } \\
\text { de Monts } \\
\text { Iguellala }\end{array}$} & Nigericeras & \multirow{2}{*}{$\begin{array}{l}\text { Formation des } \\
\text { Monts Iguellela }\end{array}$} & T3 & Calcaires à Nigericeras & \\
\hline & & $\begin{array}{l}\text { Calcaires à } \\
\text { Neolobites }\end{array}$ & $\mathrm{Tl}$ & & $\stackrel{\stackrel{a ̀}{a}}{\text { Neolobites }}$ & & $\mathrm{T} 2$ & Calcaires à Neolobites & $\mathrm{T} 1$ \\
\hline $\begin{array}{l}\text { Cénomanien } \\
\text { inférieur }\end{array}$ & & & & Groupe de Tés & 'gama & & T1 & & \\
\hline
\end{tabular}

Figure 10. Comparaison des transgressions méso-cénozoïques dans le bassin des Iullemmeden 


\section{Conclusion}

Le schéma paléogéographique du bassin des Iullemmeden au Maastrichtien supérieur et au Paléocène-Yprésien est marqué par la présence d'une mer épicontinentale, peu profonde, avec en bordure une plaine côtière marécageuse, parsemée de lagunes littorales. Dans le cadre de la présente étude, la mise en évidence d'un niveau marneux, d'environ $1 \mathrm{~m}$ d'épaisseur, riche en microfossiles de foraminifères de l'espèce Lockhartia hamei, audessus du membre de Barmou a conduit à distinguer une nouvelle transgression T6 d'âge yprésien, selon le classement des auteurs considéré. Cette nouvelle transgression ainsi identifiée apporte des nouvelles précisions sur l'histoire géologique du bassin des Iullemmeden. Ainsi, tout comme la transgression T5 du Paléocène, cette transgression T6 serait associée à la compression $\mathrm{N} 140^{\circ}$ à $\mathrm{N} 150^{\circ}$ du Paléocène-Yprésien attribuées aux contrecoups de la collision Europe-Afrique en liaison probable avec l'ouverture de l'Atlantique.

\section{References:}

1. Abdou Ali I., Konate M., Ousmane B. (2018). Lineamentary and Structural cartography of Iullemmeden Basin in the region of Dosso (South-west of Niger). IJSR, ISSN (Online) 2319-7064. Vol. 7, issue 4, 9p.

2. Alzouma K. (1982). Étude pétrologique de la série sédimentaire tertiaire du bassin de Malbaza (Niger). Thèse Doct. 3ème Cycle Univ. de Niamey et d'Orléans, 159 p.

3. Bellion Y. J. C. (1989). Histoire géodynamique post-paléozoïque de l'Afrique de l'Ouest d'après l'étude de quelques bassins sédimentaires (Sénégal, Taoudéni, Iullemmeden, Tchad), Publication occasionnelle CIFEG 17, 302 p., BRGM Orléans (France).

4. Bertrand-Sarfati J., Fabre J., Moussine Pouchkine A. (1977). Géodynamique des aires sédimentaires cratoniques: quelques exemples sahariens. Bull. Centre Rech. Explor. Prod. Elf Aquitaine, 1,1, 217-231.

5. Boudouresque L. (1980). Contribution de la paléopalynologie à la reconstitution floristique, stratigraphique et paléogéographique de la bordure occidentale du bassin des Iullemmeden au Crétacé supérieur et au Paléocène (Niger et Mali, Afrique de l’Ouest). Thèse spécialité, Univ. Orléans-Niamey, 285p.

6. Boudouresque L. Dubois D. \& Lang J. (1982). Contribution à la stratigraphie et à la paléogéographie de la bordure occidentale du bassin des Iullemmeden au Crétacé supérieur et au Paléogène (Niger et Mali-Afrique de l'Ouest). Bull. Soc. géol. Fr., t 24, n? 4, pp. 685695.pp. 105-124. 
7. Dikouma M. (1990). Fluctuations du niveau marin au Maastrichtien et au paléocène dans le bassin intracratonique des Iullemmeden (AderDoutchi, Niger). Thèse Doctorat. Univ. Dijon-Niamey, 272 p.

8. Dikouma M., Alzouma K., Lang J., Laurin B., Pascal A., Trichet J., Carbonnel G. Et Tintant H. (1987). Maastrichtien and Paleocene transgressions and régressions in West Niger; Shoreline oscillations. Terra cognita EUG IV 7, n²-3, p. 337.

9. Dikouma M., Lang J., Pascal A. (1993). Transgression Maastrichtiennes et paléogènes dans le bassin des Iullemmeden (Niger). Newsl. Stratigr., 29 (2). Berlin. Stuttgart.

10. Dubois D. \& Lang J. (1981). Étude lithostratigraphique et géomorphologique du Continental terminal et du Cénozoïque inférieur dans le bassin des Iullemmeden (Niger). Bulletin de l'I.F.A.N., t 43, série $A, n^{\circ} 1-2,42 \mathrm{p}$.

11. Dubois H. (1979). Etude géologique des formations Oolithiques ferrugineuses du bassin des Iullemmeden (République du Niger). Thèse de doctorat de $3^{\text {ème }}$ cycle, Université de Niamey et d'Orléans.

12. Faure H. (1966). Reconnaissance géologique des formations sédimentaires post-paléozoïques du Niger oriental. Mem. Bur. Rech. Geol. Minieres 47, $1^{\mathrm{e}} 629$.

13. Favreau G. (2000). Caractérisation et modélisation d'une nappe phréatique en hausse au Sahel: dynamique et géochimie de la dépression piézométrique naturelle du Kori de Dantiandou (Sud-ouest du Niger). Thèse de Doctorat, Univ. Paris Sud-Orsay, 271 p.

14. Flicoteaux R. (1982). Genèse du phosphate alumineux au Sénégal Occidental : Etape et guide de l'altération, Strasbourg, Institut de géologie, univ Louis Pasteur de Strasbourg, Mém. N67, 2231.

15. Greigert J. \& Pougnet R. (1965). Carte géologique de la république du Niger au 1/2000000. B.R.G.M., Paris.

16. Greigert J. (1966). Description des formations crétacées et tertiaires du Bassin des Iullemmeden (Afrique Occidentale). Pub. Dir. Min. -Géol. Niger, n². Mém. B.R.G.M., n³6, 234 p.

17. Guiraud R. \& Alidou S. (1981). La faille de Kandi (Bénin), témoin de rejeu fini-crétacé d'un accident majeur à l'échelle de la plaque africaine. C.R. Accad. Sci. Paris, 293, pp. 779-782.

18. Hanon M. (1984). Cartes géologiques au 1/100.000 de l'Ader Doutchi (16 feuilles). DRGM Niger. AGCD Belgique.

19. Joulia F. (1963). Carte géologique de reconnaissance de la bordure sédimentaire occidentale de l'Aïr au 1/500 000. Éditions du BRGM, Orléans, France.

20. Kilian C. (1931). Des principaux complexes continentaux du Sahara. C.R. Soc.géol.Fr., pp. 109-111. 
21. Kogbe C. A. \& Lang. J. (1994). Bassin intracratonique des Iullemmeden. Afrique de l'Ouest. Africa geoscience review, vol. 1, $\mathrm{n}^{\circ}$ 2/3, 6 p.

22. Laouali Idi K. \& Konate M. (2019). Extraction de réseaux de fractures en milieu sédimentaire par les techniques de la télédétection intégrant une analyse statistique et structurale : cas des formations mésocénozoïques du bassin des Iullemmeden, Niger Central. Afrique Science 15(5) (2019) 39 - 53.15p.

23. Laouali Idi K. (2020). Etudes sédimentologique, paléontologique, paléogéographique et structurale des dépôts d'âge maastrichtien à yprésien du bassin des Iullemmeden (Niger central). Thèse de Doctorat Unique. Université de Niamey, 173 p.

24. Mathey B., Lang J., Alzouma K. \& Pierre C. (1990). Nouvelle interprétation des premiers mouvements marins du Crétacé supérieur dans la partie orientale du bassin des Iullemmeden (Niger, Afrique de l'Ouest). 15ème Coll. Géol. afr. Nancy, Pub. occas. , pp. 104.

25. Miko I. (1999). Dynamique sédimentaire des formations détritiques et ligniteuses du Continental terminal dans le bassin des Iullemmeden (Niger). Thèse de Doctorat Université Abdou Moumouni Faculté des Sciences-Département de Géologie Niamey, 328 p.

26. Petters S. W. (1978). Maastrichtian-Paleocene foraminiferal from North-West Nigeria and their paleogeography. Acta Paleontologica Polonica, 23, n², 131-152.

27. Taquet P. (1976). Les découvertes récentes de Dinosaures du Jurassique et du Crétacé en Afrique, au Proche Orient et en Inde. Mém. Soc. Géol. Fr. h. s. n8, pp. 325-330. Terminal et du Cénozoïque inférieur dans le bassin des Iullemmeden (Niger). Bull.I. F. terminal et du cénozoïque inférieur dans le bassin des lullemmeden, Dakar- IFAN.

28. Wright L. I., Branchet M., \& Alisso I. (1993). Notice explicative de la carte géologique du bassin houillier d'Anou Araren / Solomi. Ministère des Mines et de l'Énergie, Niger, 20 p. 